\title{
Análisis discriminante aplicado a los grupos sexuales de Potimirim mexicana, camarón hermafrodita protándrico
}

\section{Discriminant analysis applied to the sexual groups of Potimirim mexicana, a protandric hermaphrodite shrimp}

\author{
Ma. del Pilar Alonso-Reyes ${ }^{1 *}$, José Luis Bortolini-Rosales ${ }^{2}$ y Fernando Álvarez ${ }^{3}$ \\ ${ }^{1}$ Departamento de Matemáticas, Facultad de Ciencias, Universidad Nacional Autónoma de México. Ciudad Universitaria, Del. Coyoacán 04510 \\ México, D. F., México. \\ ${ }^{2}$ Departamento de Biología Comparada, Facultad de Ciencias, Universidad Nacional Autónoma de México. Ciudad Universitaria, Del. Coyoacán \\ 04510 México, D. F., México. \\ ${ }^{3}$ Colección Nacional de Crustáceos, Instituto de Biología, Universidad Nacional Autónoma de México. Ciudad Universitaria, De.l Coyoacán 04510 \\ México D. F., México. \\ *Correspondencia: sarpar@att.net.mx
}

\begin{abstract}
Resumen. Se analizó una población del camaroncito de río Potimirim mexicana obtenida del río Máquinas en el estado de Veracruz, México. Esta especie presenta una distribución de tallas por sexo que sugiere que se trata de una especie con hermafroditismo secuencial. Se aplicó el método de análisis discriminante para establecer los subgrupos que conforman la población, dando como resultado 3 clases: organismos sexualmente indeterminados, machos y hembras. En el subgrupo de los machos se establecieron 3 conjuntos, con base en el tamaño del appendix masculina. Mediante este análisis se confirmó que P. mexicana es una especie hermafrodita secuencial.
\end{abstract}

Palabras clave: Potimirim mexicana, hermafroditismo, análisis discriminante, análisis de componentes principales.

\begin{abstract}
A population of the river shrimp Potimirim mexicana from the Máquinas River in Veracruz, Mexico, was analyzed. The pattern of size distribution by sex in this species suggests that it is a sequential hermaphrodite. A discriminant analysis method applied to establish the subgroups that are part or the population showed the existence of 3 classes: sexually undifferentiated organisms, males and females. Within the male subgroup 3 types or organisms were established depending on the length of the appendix masculina. With this analysis it is confirmed that $P$. mexicana is a species with sequential hermaphroditism.
\end{abstract}

Key words: Potimirim mexicana, hermaphroditism, discriminant analysis, principal component analysis.

\section{Introducción}

Dentro de la reproducción en crustáceos existen 2 tipos de hermafroditismo: el secuencial y el simultáneo. Ejemplos del primero son los que se presentan en el género Lysmata o en el isópodo Gnorimosphaeroma oregonense, en el que se ha observado un hermafroditismo secuencial protogíneo (Heather et al., 1994; Bauer y Holt, 1998; Fiedler, 1998; Bauer, 2000). En la protandria, un individuo sexualmente indiferenciado madura en primera instancia como macho y posteriormente se transforma en hembra (Charnov, 1979). Así, los organismos más pequeños son sexualmente indiferenciados; un segundo subconjunto de tallas intermedias corresponde a los machos, y el tercero y último grupo, con los individuos más grandes,

Recibido: 26 noviembre 2009; aceptado: 26 abril 2010 corresponde a las hembras.

En los litorales mexicanos se han observado organismos del género Potimirim; P. glabra se distribuye en las costas del Pacífico, desde el sur del país hasta Centroamérica y $P$. mexicana en las costas del golfo de México, desde el río Soto La Marina en Tamaulipas hasta el río Grijalva en Tabasco (Villalobos, 1959). En estas especies, el dimorfismo sexual es evidente en la distribución de tallas. Los organismos sexualmente indiferenciados son los que tienen un largo total (LTo) de 2.0 - $8.0 \mathrm{~mm}$; los machos miden de 5.0 - $8.0 \mathrm{~mm}$, presentando un appendix masculina situado sobre el segundo par de pleópodos y las hembras, de 10.0 - $26.0 \mathrm{~mm}$ y en algunas poblaciones se ha registrado la presencia de hembras desde los $6.2 \mathrm{~mm}$ (Martínez, 2003). En el caso de los machos de P. mexicana, la madurez sexual se adquiere a los $8.0 \mathrm{~mm}$ de LTo y en las hembras a los $11.0 \mathrm{~mm}$ (Luna, 1989). 
Para establecer relaciones morfométricas en camarones se han utilizado variables como: el largo del caparazón, el largo total, el largo del abdomen y el peso húmedo (Primavera et al., 1998; Dall et al., 1990); sin embargo, estas variables pueden también ser analizadas por intervalos de tamaño para determinar cómo cambian en relación con el estadio de vida (Chow y Sandifer, 1991). En este estudio se utilizó la función de análisis discriminante con el objetivo de estimar ecuaciones que sean aplicadas para clasificar a los individuos de la población en las diferentes categorías o subgrupos, para lo cual se emplearon las variables morfométricas. La clasificación trata de subdividir la población de la manera más precisa posible, considerando disminuir el error de que un elemento pertenezca a más de una categoría. Así, se pretende que a través de una técnica estadística se pueda discernir acerca de los diferentes estadios de madurez sexual que se presentan en camarones del género Potimirim.

\section{Materiales y métodos}

Se realizaron 5 recolecciones de $P$. mexicana en el río Máquinas en la región de Los Tuxtlas, Veracruz, México (Fig. 1), entre abril de 2003 y mayo de 2004, obteniéndose 191 organismos de diferentes tallas. Se les tomaron 8 medidas del cuerpo: largo total (LTo), largo del caparazón (LC), diámetro del ojo (DO), largo del telson (LT), largo del mero (LMe) y del carpo (LCa) del tercer pereiópodo y largo del segundo segmento del primer (L1erPl) y segundo pleópodos (L2doPl). Para el caso de los machos se tomaron

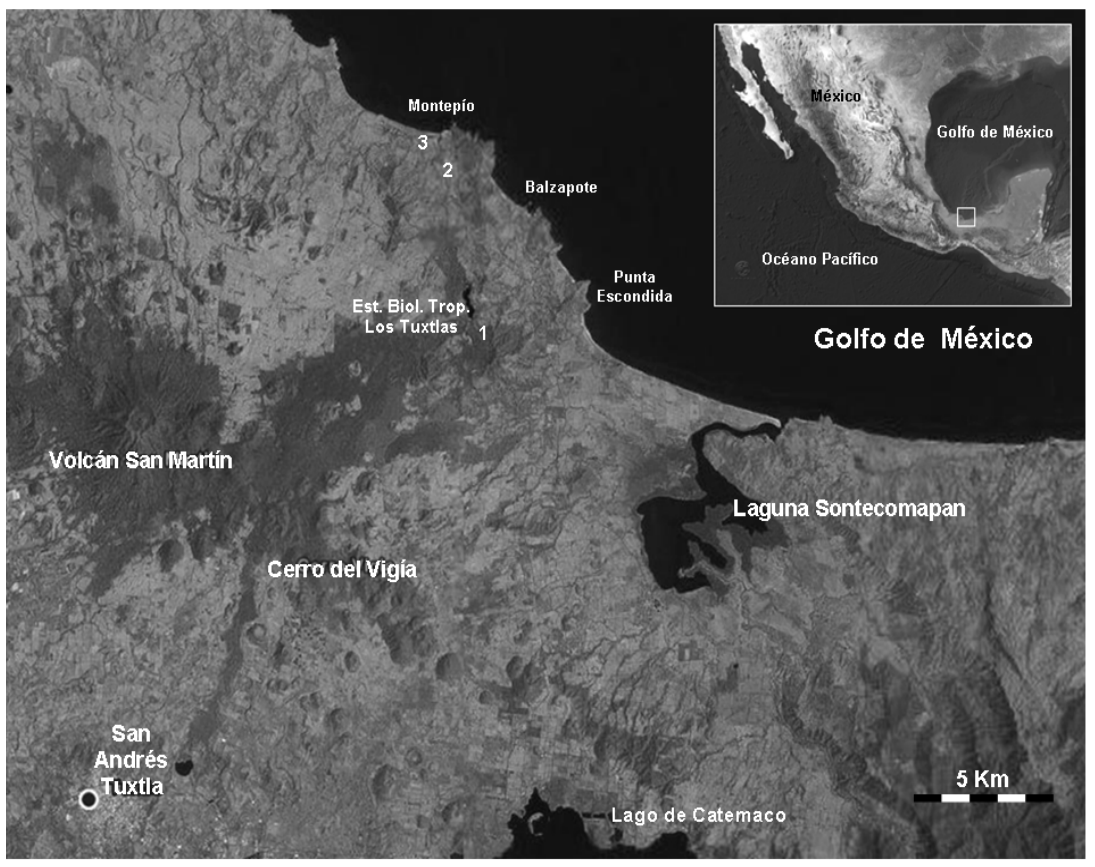

2 medidas adicionales, el largo (LAM) y ancho (AAM) del appendix masculina (Fig. 2).

Para el análisis se utilizó la técnica de discriminante con el total de organismos, manejando la clasificación $a$ priori del sexo. En la técnica de discriminación se desea pronosticar la pertenencia de una observación particular a una clase $\left(\Pi_{1}, \Pi_{2}, \ldots, \Pi_{g}\right)$, con base en un conjunto de variables predictoras $(X)$ (Anderson, 1984; Dillon y Goldstein, 1984; Krzanowski, 1996; Seber, 1984). El objetivo básico es hacer una regla de clasificación que permita pronosticar la población de la cual es más probable que provenga una observación, esto es:

Considerando $g$ grupos $\Pi_{1}, \Pi_{2}, \ldots, \Pi_{g}, g \geq 2$ y suponiendo para cada $\Pi_{j}$, una función de densidad de $f_{j}(X)$ sobre el espacio de dimensión $p\left(R^{P}\right)$, de tal forma que si un individuo proviene de la población $\Pi_{j}$ , tiene función de probabilidad $f_{j}(X)$. Entonces el objetivo es asignar un individuo a uno de estos $g$ grupos de acuerdo con sus mediciones $X$. Se necesita una regla discriminante $d$ correspondiente a una partición de regiones (subgrupo)

$$
\text { de } R^{P},\left(R_{1}, R_{2}, \ldots, R_{g} ; \bigcup_{i=1}^{g} R_{i}=R^{P}\right) \text {. }
$$

La regla $d$ está definida por:

$$
\text { Asigna } X \text { a } \Pi_{j} \text { si } X \in R_{j} \text {, para } j=1,2, \ldots, g \text {. }
$$

La discriminación será más exacta si $\Pi_{j}$ tiene una gran probabilidad concentrada en $R_{j}$ para $j$. El proceso de asignación se puede observar en la figura 3 .

Para el análisis del appendix masculina se utilizó primero la técnica de componentes principales, para determinar qué posibles grupos se podían formar y después se revisó la separación en conjuntos a través del análisis discriminante.

El análisis de componentes principales (Anderson, 1984; Dillon y Goldstein, 1984; Krzanowski, 1996; Seber, 1984) tiene básicamente 2 objetivos: reducir la dimensionalidad del conjunto de datos, e identificar nuevas variables significativas subyacentes.

La idea es describir la variación deunconjuntodedatosmultivariados

Figura 1. Mapa del área de estudio en la región de Los Tuxtlas, en el sur del estado de Veracruz, México. 1, 2 y 3, sitios de colecta de Potimirim del río Maquinas. 


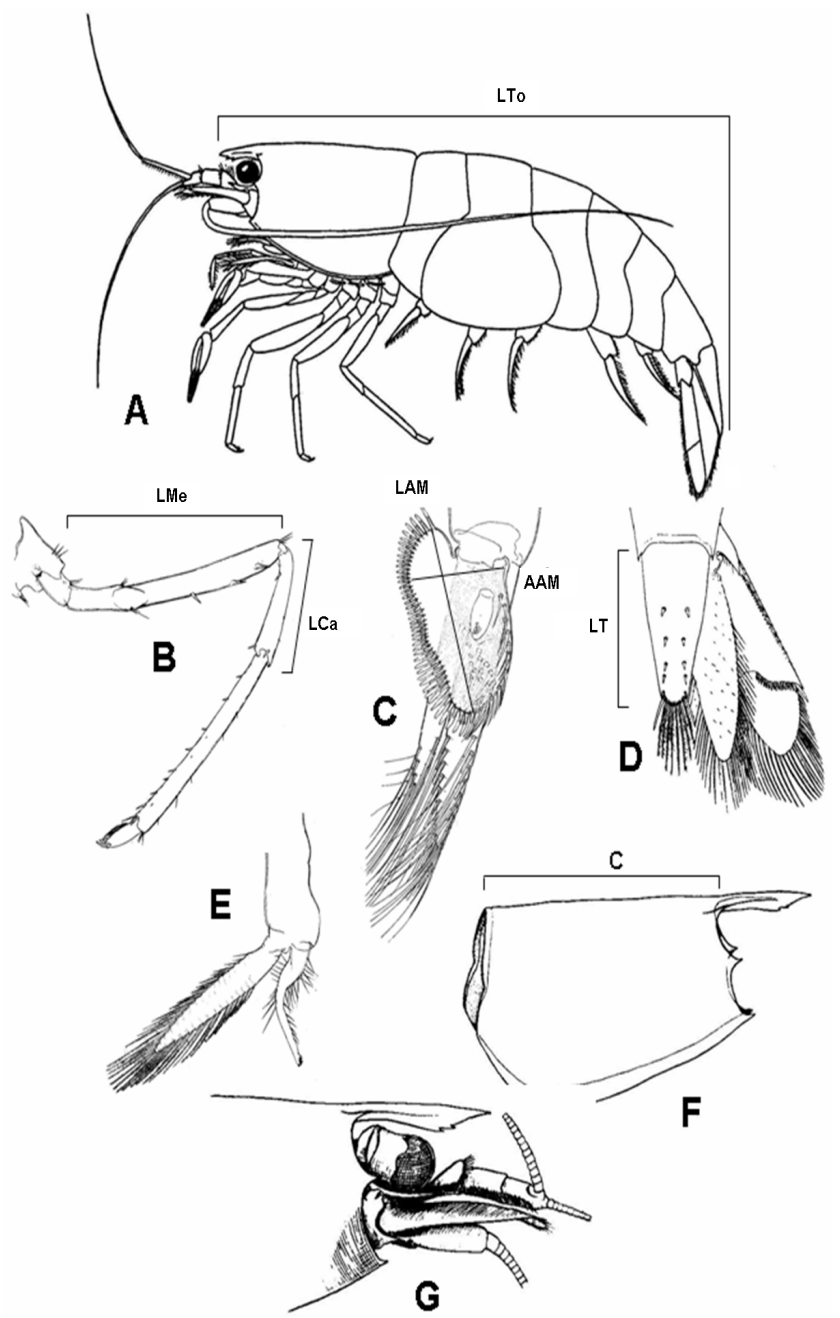

Figura 2. Morfometrías de Potimirim mexicana. A, largo total (LTo); B, quinto pereiópodo (LMe, largo del mero; LCa, largo del carpo); C, Appendix masculina sobre el segundo pleópodo (LAM, largo; AAM, ancho); D, telson (LT); E, primer pleópodo (L1Pl, L2Pl); F, largo del caparazón (LC); G, rostro ubicando el apéndice ocular. A, tomado de Fryer (1977); B-G, tomados de Villalobos (1959).

$X^{\prime}=\left(X_{1}, \ldots, X_{p}\right)$ en términos de un agregado de variables no correlacionadas, cada una de las cuales es una combinación lineal (suma de las variables) particular de las originales. Las nuevas variables (componentes principales) son derivadas en orden decreciente de importancia; por ejemplo, la primera componente principal aporta tanto como es posible de la variación de los datos originales, y así sucesivamente. En este sentido, cuando se toman las primeras componentes se tiene una gran explicación de la variabilidad de los datos originales y se habrá reducido la dimensionalidad.

Sea $X^{\prime}=\left(X_{1}, \ldots, X_{p}\right)$ un vector de observaciones que tiene matriz de varianza-covarianza $\Sigma$ (Dillon y Goldstein, 1984:b27). La primera componente, $Y_{(i)}$, será una combinación lineal de las variables originales, de tal forma que ésta tenga la máxima varianza posible; por ejemplo, $Y_{(1)}$ la primera componente, será:

$$
Y_{(1)}=\gamma_{1}^{\prime} X \text { (la primera combinación lineal) }
$$

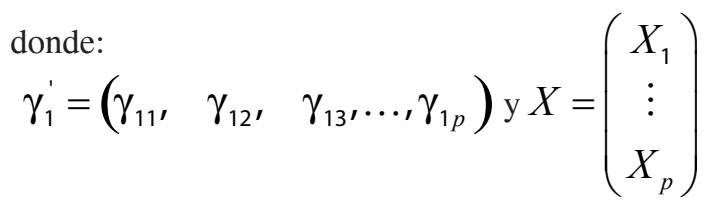

de esta forma:

$$
\operatorname{Var}\left(Y_{(1)}\right)=\operatorname{Var}\left(\gamma_{1}^{\prime} X\right)=\gamma_{1}^{\prime} \sum \gamma_{1}
$$

Considerando que lo que se pretende es maximizar la varianza explicada $\left(\gamma_{1} \sum \gamma_{1}\right)$, sujeta a la condición $\gamma_{1}^{\prime} \gamma_{1}=1$; permite generar el método para cada componente principal, determinando un sistema de $p$ ecuaciones de la forma:

$$
(\Sigma-\lambda I) \gamma=\underline{0}
$$

donde $I$ es la matriz identidad y $\underline{0}$ es el vector de ceros. La solución implica la obtención de valores $\lambda_{i}$ que son la $\operatorname{Var}\left(Y_{i}\right)=\lambda_{i}$ y que entre ellas guardarán la relación:

$$
\lambda_{1} \geq \lambda_{2} \geq \ldots \geq \lambda_{p}
$$

La determinación del número de componentes a utilizar permite generar una gráfica que muestra todas las variables de estudio de los organismos y determina a priori nubes de puntos que implican grupos con características similares. Esto es lo primero que se hizo con las variables de los organismos machos, y posteriormente se aplicó la técnica de análisis discriminante para determinar si la clasificación había sido correcta.

\section{Resultados}

Con la totalidad de organismos se hace una discriminación utilizando la separación hecha a priori del sexo y las variables LC, DO, LT, L1erPl, L2doPl, LMe, LCa y LTo, obteniendo 3 subgrupos: organismos sexualmente indeterminados, machos y hembras (Figs. 4, 5).

El análisis discriminante mostró una primera clasificación que se da en el Cuadro 1, la cual indicó que se tienen 17 hembras observadas y que la combinación de las 8 variables de análisis indicaron que realmente son 


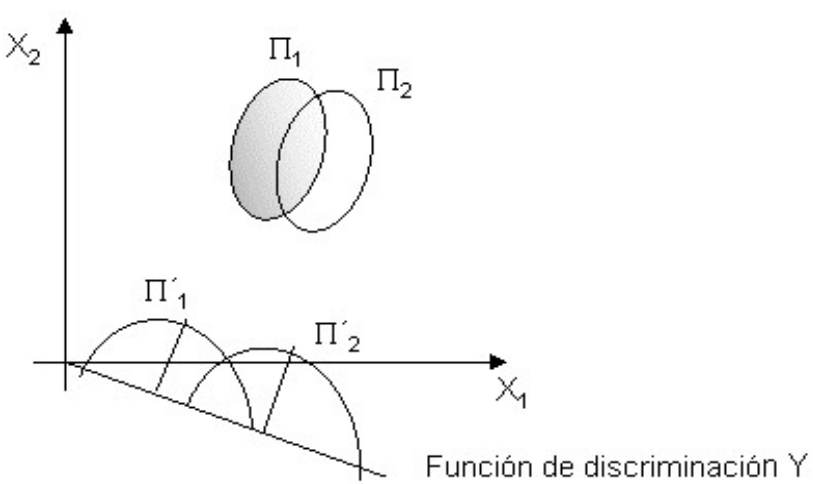

Figura 3. Proceso de discriminación entre la nube de puntos y la regla de separación. Tomado de Dillon y Goldstein, 1984.

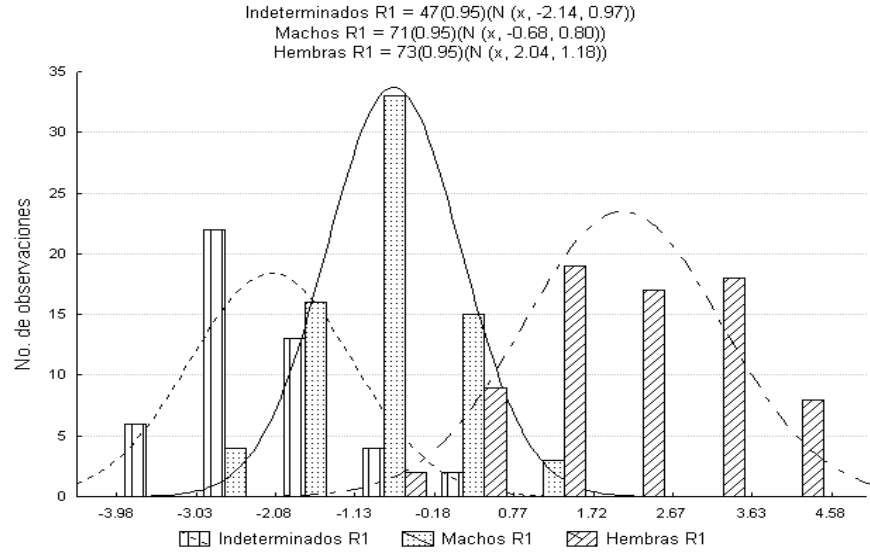

Figura 4. Discriminación de las 3 poblaciones por sexos.

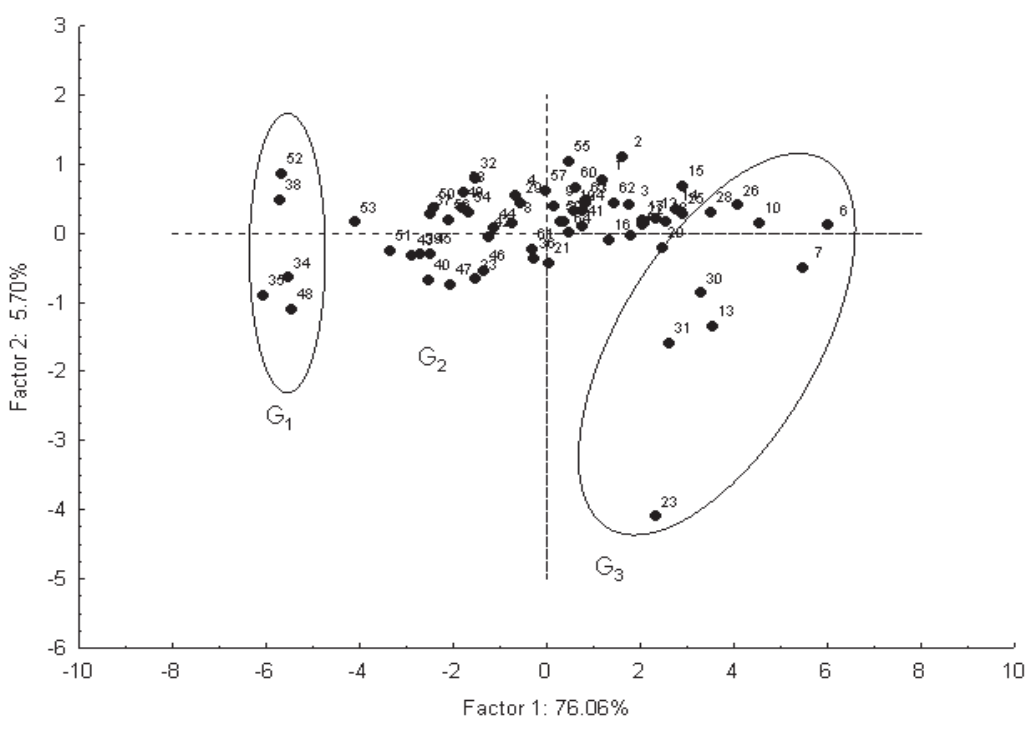

Figura 5. Proyección de los casos, utilizando los componentes principales. que los subgrupos se traslapan entre sí, con la intersección de las colas de las distribuciones de cada subgrupo, e indica cómo existen elementos que están correctamente clasificados y no tienen posibilidad de ser clasificados en otro subgrupo.

El siguiente paso fue analizar los machos (64 organismos), los cuales fueron tomados de la clasificación a priori por sexo. Se consideraron las 8 variables anteriores incluyéndose el largo y ancho del appendix masculina. No se tenía una clasificación de antemano de estos organismos basada en esta estructura, por lo que se utilizó la técnica de componentes principales para poder disminuir la dimensionalidad de las variables y lograr

machos; lo mismo sucedió con 4 hembras que realmente fueron indeterminadas; en el caso de los machos se tuvo que 9 de ellos fueron hembras y 6 indeterminados; por último, para el caso de los organismos indeterminados se obtuvo 1 que fue hembra y 5 machos.

Se analizaron las clasificaciones incorrectas y se corrigieron los errores, y nuevamente se aplicó el análisis discriminante dando como resultado lo observado en el Cuadro 2 con un $92.67 \%$ de clasificación correcta, el cual es un porcentaje muy alto. La nueva función de discriminación indicó que solamente 14 casos fueron clasificados incorrectamente, 5 hembras que fueron machos, 2 hembras que fueron indeterminados, 4 machos pasaron a indeterminados y 3 indeterminados a machos.

La clasificación que produjo la técnica de análisis discriminante se muestra en la figura 3, donde se observa una clasificación adecuada. En la figura 5, se muestran los primeros 2 componentes principales y los organismos machos. Puede observarse que se formaron 3 subgrupos, el primero (G1) con 5 organismos, el segundo (G2) con 49 y el tercero (G3) con 10.

Se continuó con el examen aplicando el análisis discriminante con el fin de verificar si era correcta esta categorización de los machos, y se obtuvo el $100 \%$ de clasificación correcta. Se procedió a ordenar esos grupos y verificar las medidas descriptivas que daban en todas las variables, por lo que puede decirse que los organismos pertenecientes a G1 son aquellos cuyo appendix masculina es en promedio el de mayor tamaño; $\mathrm{G} 2$, representa los de tamaño medio y finalmente G3 los de menor tamaño (Fig. 6).

Para analizar el ancho y largo de esta estructura, en la 
Cuadro 1. Clasificación con la técnica de discriminante y la clasificación a priori por sexo

\begin{tabular}{lrrrr}
\hline & $\begin{array}{c}\text { \% clasif. } \\
\text { correcta }\end{array}$ & Hembra & Macho & $\begin{array}{r}\text { Indeter- } \\
\text { minado }\end{array}$ \\
\hline Hembra & 75.00000 & 63 & 17 & 4 \\
Macho & 76.56250 & 9 & 49 & 6 \\
Indeterminado & 86.04651 & 1 & 5 & 37 \\
Total & 78.01047 & 73 & 71 & 47 \\
\hline
\end{tabular}

Cuadro 2. Clasificación con la técnica de discriminante y la clasificación corregida con base en el sexo

\begin{tabular}{lccrc}
\hline & $\begin{array}{c}\text { \% clasif. } \\
\text { correcta }\end{array}$ & Hembra & Macho & $\begin{array}{r}\text { Indeter- } \\
\text { minado }\end{array}$ \\
\hline Hembra & 90.41096 & 66 & 5 & 2 \\
Macho & 94.36620 & 0 & 67 & 4 \\
Indeterminado & 93.61702 & 0 & 3 & 44 \\
Total & 92.67016 & 66 & 75 & 50 \\
\hline
\end{tabular}

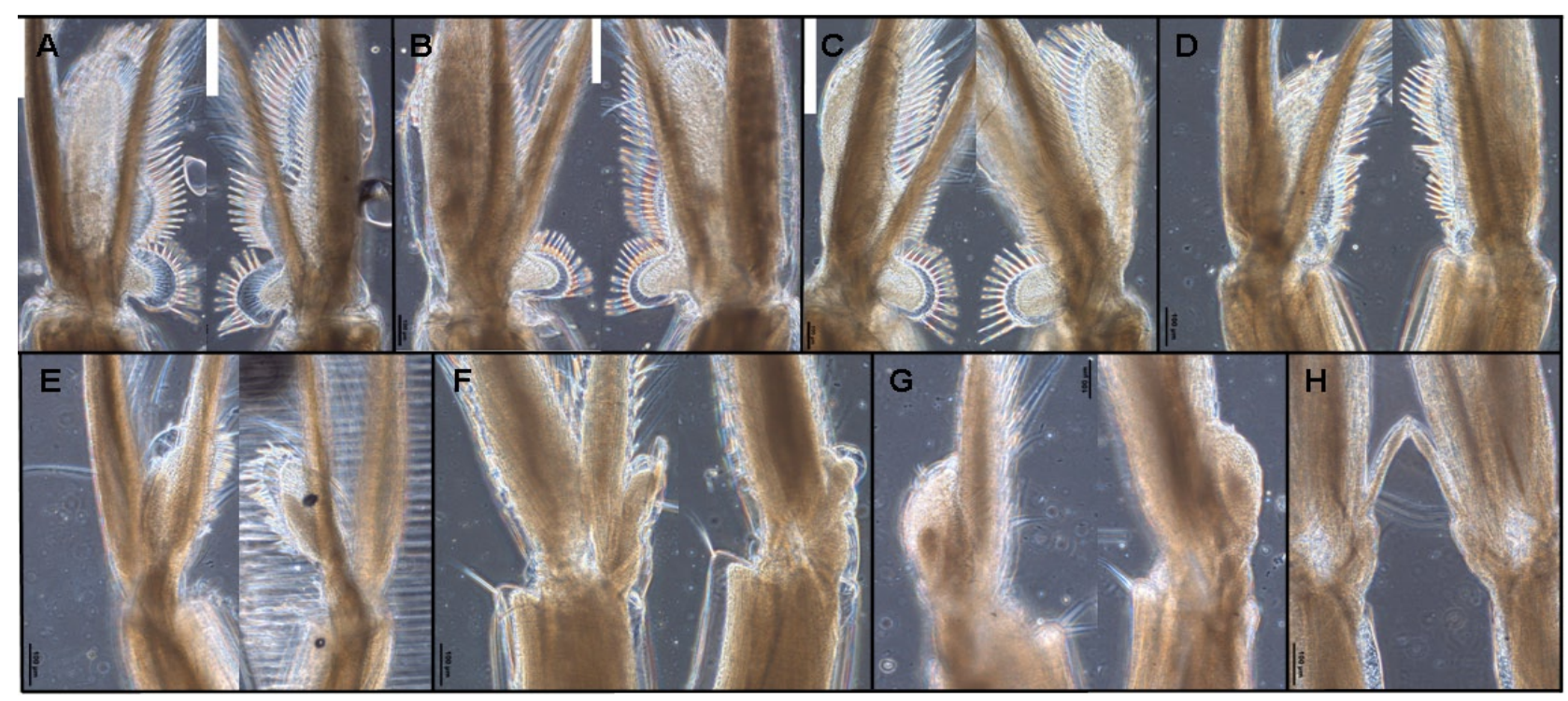

Figura 6. Appendix masculina perteneciente a los 3 diferentes grupos mostrados en la figura 5. A-C, estructuras de mayor dimensión, subgrupo de la izquierda; D-E, de dimensiones medias, subgrupo intermedio y F-H, de dimensiones menores, subgrupo de la derecha.

figura 7 se muestran las 2 variables a 2 escalas diferentes. Puede observarse que ambas revelan un comportamiento parecido; G1 consta de 5 organismos que son los que tienen en promedio el appendix masculina más grande, G2 formado por 49 elementos con medidas estructurales medias y por último G3 constituido por 10 organismos que muestran una estructura en promedio más pequeña, corroborando los resultados obtenidos en los componentes principales.

\section{Discusión}

Potimirim mexicana es un camarón átyido que mostró un patrón de tallas por sexo que sugiere que se trata de una especie que pasa de indiferenciado a macho y finalmente a hembra. Al no haber un gran traslape en las tallas de los
3 estadios que presentó (indiferenciado, macho y hembra) no existe otra explicación para obtener este patrón que la de que se trate de un hermafroditismo secuencial. Aun cuando ésta es con toda probabilidad la explicación correcta, los estudios histológicos que sin lugar a dudas la demostrarían están en proceso y no han sido publicados. Como una herramienta adicional que describa el fenómeno biológico se utilizó el análisis discriminante para poner a prueba si estadísticamente se podía detectar este tipo de maduración.

El análisis discriminante demostró ser una técnica útil para diferenciar entre los 3 diferentes estadios de $P$. mexicana. De esta manera se exhibió un método estadístico que ayuda a describir un fenómeno biológico con gran precisión. Potimirim mexicana pasa por 3 estadios que pueden caracterizarse por su largo total con cierto grado de traslape. Cuando se hacen correcciones y se corre el 


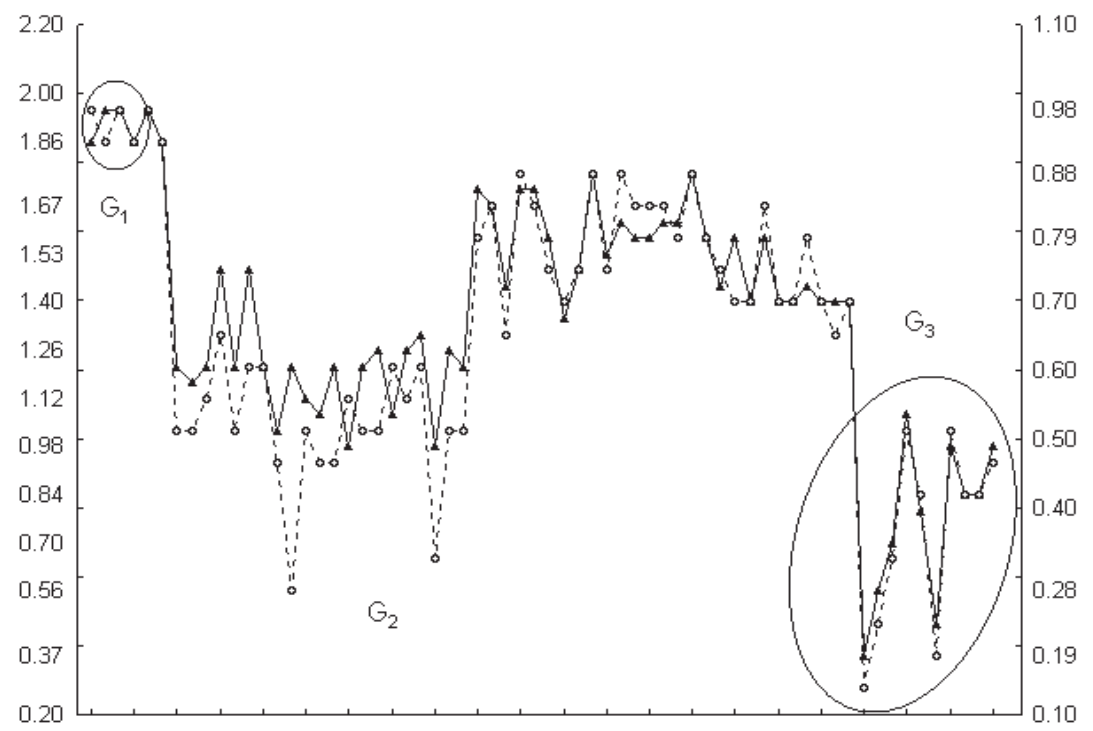

Figura 7. Comportamiento del largo (LAM) y ancho (AAM) del appendix masculina.

análisis nuevamente, la precisión de la clasificación aumenta de manera considerable, hasta llegar al $100 \%$. En la naturaleza es posible que el traslape esté dado por diferencias estacionales en el crecimiento o por variaciones dadas por la localización geográfica.

\section{Agradecimientos}

A Carmen Hernández por su apoyo en el trabajo en campo. Fernando Álvarez agradece el apoyo recibido a través del proyecto IN203906-3 financiado por el programa PAPIIT-UNAM.

\section{Literatura citada}

Anderson, T. W. 1984. An introduction to multivariate statistical analysis, segunda edición. John Wiley, Hoboken, New Jersey p. 451-479 y 228-241.

Bauer, R. T. 2000. Simultaneous hermaphroditism in caridean shrimps: a unique and puzzling sexual system in the Decapoda. Journal of Crustacean Biology 20 (special issue):116-128.

Bauer, R. T. y G. J. Holt. 1998. Simultaneous hermaphroditism in the marine shrimp Lysmata wurdemanni (Caridea: Hippolytidae): an undescribed sexual system in the decapod Crustacea. Marine Biology 132:223-235.

Charnov, E. L. 1979. Natural selection and sex change in pandalid shrimp: test of a life-history theory. American Naturalist 113:715-734

Chow, S. y P. A. Sandifer. 1991. Differences in growth, morphometric traits, and male sexual maturity among Pacific white shrimp, Penaeus vannamei, from different commercial hatcheries. Aquaculture 92:165-179.

Cooley, W. W. y P. R. Lohnes. 1971. Multivariate data analysis. John Wiley, Hoboken, New Jersey. 364 p.
Dall, W., B. J. Hill, P. C. Rothlisberg y D. J. Staples. 1990. The biology of the Penaeidae. Advances in marine biology. Academic, London. 489 p.

Dillon, W. y M. Goldstein. 1984. Multivariate analysis. Methods and applications. John Wiley, Hoboken, New Jersey. 587 p.

Fiedler, G. C. 1998. Functional, simultaneous hermaphroditism in female-phase Lysmata amboinensis (Decapoda: Caridea: Hippolytidae). Pacific Science 52:161-169.

Heather, J. B., A. R. Timothy y R. W. Davies. 1994. Protogynous sex change in the intertidal isopod Gnorimosphaeroma oregonense (Crustacea: Isopoda). Biological Bulletin 187:99-111.

Krzanowski, W. J. 1996. Principles of multivariate analysis. A user's perspective. Oxford Statistical Science Series, New York. 608 p.

Luna, M. M. L. 1989. Aspectos biológicos de Potimirim mexicana bajo la influencia estuarina del río la Antigua, Veracruz. Tesis, Universidad Veracruzana, Xalapa Veracruz. 23 p.

Martínez, M. 2003. Contribución al conocimiento de la biología y ecología de Potimirin glabra Kingsley (Decapoda, Atyidae) en el río Coyuca, Guerrero. Tesis Maestría Facultad de Ciencias, Universidad Nacional Autónoma de México, México, D.F. 64 p.

Primavera, J. H., F. D. Parado-Estepa y J. L. Lebata. 1998. Morphometric relationship of length and weight of the giant tiger prawn Penaeus monodon according to life stage, sex and source. Aquaculture 164:67-75.

Seber, G. A. F. 1984. Multivariate observations, second edition. John Wiley, Hoboken, New Jersey. 686 p.

Villalobos, A. 1959. Contribución al conocimiento de los Atyidae de México. II (Crustacea, Decapoda). Estudio de algunas especies del género Potimirim (=Ortmannia), con descripción de una especie nueva en Brasil. Anales del Instituto de Biología, Universidad Nacional Autónoma de México, Serie Zoología 30:269-330. 